\title{
Effects of CSs for food and water upon rats barpressing for different magnitudes of food reinforcement
}

\author{
RICHARD M. WIELKIEWICZ \\ University of Hawaii, Honolulu, Hawaii 96822
}

\begin{abstract}
Forty-eight albino rats were first exposed to randomly intermixed, noncontingent presentations of one pellet, six pellets, and water. For each of three groups, a CS+ was paired with one of the reinforcers, while the other two reinforcers and a CS- were presented alone. Then half the subjects experienced a six-pellet reward and half experienced a one-pellet reward for leverpresses during a light $\mathrm{SD}^{\mathrm{D}}$. Finally, on test trials, the CS+ or CS- was compounded with the light. Latencies on test trials indicated that responding for the one-pellet reward was significantly more disrupted by a CS+ for six pellets than by the CS+ for one pellet or the CS+ for water. Responding for the six-pellet reward was disrupted by the CS+ for one pellet somewhat more than by the CS+ for six pellets or water, but not significantly so. This pattern of results is partially consistent with an associative model of transfer and appears to contradict a motivational account of such effects.
\end{abstract}

Both motivational (e.g., Rescorla \& Solomon, 1967; Spence, 1956) and associative (e.g., Trapold \& Overmier, 1972) properties have been attributed to the classically conditioned state hypothecized to mediate control of previously learned instrumental responses by a classical CS. Motivational accounts have assumed that the tendency of an animal to perform a response to the test stimulus (CS) is partially determined by the incentive properties (cf. Spence, 1956) of the mediator. Accordingly, an instrumental response should be facilitated by a CS previously paired with a reinforcer, because a mediator is elicited which increases total incentive motivation, which in turn multiplies the habit strength of the test response. On the other hand, the associative model assumes that CS effects result from associative properties of the mediator which are established during instrumental training. First, stimulus feedback from the mediator or "expectancy" of reward, classically conditioned to environmental cues during instrumental training, becomes part of the stimulus complex controlling the behavior. Then presentation of a CS which elicits this same or a similar mediator tends to exert some stimulus control over the in-

\footnotetext{
This paper is part of a dissertation submitted to the Graduate Division of the University of Hawaii in partial fulfillment of the requirements for the $\mathrm{PhD}$ degree in psychology. My thanks to J. G. Carlson for his guidance during the conduct of this research and to J. B. Overmier, J. Patterson, and M. A. Trapold for their comments upon early drafts of this paper. Preparation of this manuscript was supported by a grant to the Center for Research in Human Learning, University of Minnesota, from NICHHD (T36-HD-07151). Reprint requests should be sent to Richard M. Wielkiewicz, Room 205, Elliott Hall, 75 East River Road, Minneapolis, Minnesota 55455.
}

strumental response because the behavior was previously learned in the mediator's presence.

Studies comparing the transfer effects of CSs for different magnitudes of food have tentatively supported the associative interpretation and contradicted the motivational view. Carlson (1976) paired one auditory stimulus $\left(\mathrm{CS}_{\mathrm{lo}}^{+}\right)$with a single food pellet and another auditory stimulus $\left(\mathrm{CS}_{\mathrm{hi}}^{+}\right)$with eight food pellets. Subsequently, the subjects were rewarded with a single food pellet for pressing a lever at the onset of a light. When the auditory stimuli were compounded with the light on selected test trials, latencies to $\mathrm{CS}_{\mathrm{lo}}^{+}$were significantly shorter than latencies to $\mathrm{CS}_{\mathrm{hi}}^{+}$. Since instrumental training had previously brought the response under stimulus control of the one-pellet mediator, shorter latencies to $\mathrm{CS}_{\mathrm{lo}}^{+}$are in accord with the associative view. The motivational view would have predicted the opposite outcome because more incentive should have been elicited by $\mathrm{CS}_{\mathrm{hi}}^{+}$than by $\mathrm{CS}_{\mathrm{lo}}^{+}$. Unfortunately, as Carlson noted, his experiment could not be regarded as conclusive, because $\mathrm{CS}_{\mathrm{hi}}^{+}$could have disrupted the instrumental behavior independent of the conditions of instrumental reward by, for instance, inducing longer visits to the food hopper. A less ambiguous experiment would vary UCS magnitude and instrumental reward magnitude orthogonally.

Capaldi, Hovancik, and Friedman (1976) employed an appropriate design in two experiments. They compounded CSs for high- and low-magnitude reinforcers with the discriminative stimulus for an instrumental response established with either a highmagnitude reward or a low-magnitude reward. Experiment 1 consisted of one test phase conducted 
magnitude reward or a low-magnitude reward. Experiment 1 consisted of one test phase conducted while the instrumental response was reinforced, followed by a second test phase conducted during extinction. Experiment 2 tested the effect of the CSs only during extinction. In all three test phases, the effects of the CSs on a response established with a low-magnitude reward were consistent: latencies to a $\mathrm{CS}_{\mathrm{hi}}^{+}$were significantly longer than latencies to a $\mathrm{CS}_{\text {lo }}^{+}$. This is in accord with the effects obtained by Carlson (1976) and supports the associative view of transfer as opposed to the motivational view.

Capaldi et al. did not obtain consistent results when the CSs were presented to subjects trained to respond for high-magnitude reward. In two test phases, the latencies to $\mathrm{CS}_{\mathrm{hi}}^{+}$did not differ significantly from latencies to $\mathrm{CS}_{\text {lo }}^{+}$. However, during the extinction phase of Experiment 1, subjects showed significantly longer latencies to $\mathrm{CS}_{l_{0}}^{+}$than $\mathrm{CS}_{\mathrm{hi}}^{+}$, a result which could be interpreted as supporting the associative model. Unfortunately, some ambiguity arises when response latencies to the discriminative stimulus (SD) are also considered. The problem for the associative model is that latencies to $\mathrm{CS}_{\mathrm{hi}}^{+}$differed significantly from those to the SD alone while latencies to $\mathrm{CS}_{\mathrm{lo}_{\mathrm{o}}}^{+}$did not. If the animals were responding to the associative properties of the CSs, then $\mathrm{CS}_{\text {lo }}^{+}$instead of $\mathrm{CS}_{\mathrm{hi}}^{+}$ should have produced latencies significantly different from the $\mathrm{SD}$ for a high-magnitude reward.

This does not imply that the associative model is lost, because the pattern of effects during the reinforced test phase of the Capaldi et al. experiment were in the direction predicted by an associative model. Unfortunately, these effects were not all statistically significant. However, the CSs received additional training in the reinforced test phase when contingent baseline reinforcement was delivered during CS presentations. As suggested by Capaldi et al., it is possible that the additional learning could have attenuated the differences between the mediators established during the pairing phase, since both CSs were paired with the same instrumental reinforcer. Another potential problem with this experiment was that transfer effects of the CSs were compared within subjects, rather than against controls for nonassociative effects of the pairing procedure.

In some respects, the present experiment was similar to that of Capaldi et al. (1976). However, in the present experiment, test trials were never rewarded (although non-test trials were always reinforced), so that no additional conditioning except extinction was possible after the pairing phase. In addition, transfer effects were measured by comparing latencies of a CS paired with food to latencies of a CS never paired with food (CS-), thus controlling for nonassociative effects of the US presentations. Finally, the experiment also tested transfer effects of
CSs paired with a third (possibly orthogonal) reinforcer, water, upon baselines reinforced with highand low-magnitude food. Since the physical properties of water (and, presumably, its associated mediator) are very different from those of food, an associative model would predict longer latencies to CSs paired with water than to CSs paired with either food magnitude. Therefore, the present experiment provides for stronger tests of the competing hypotheses that purport to explain transfer of control.

\section{METHOD}

\section{Subjects}

Forty-eight male albino rats (Simonson Laboratories), 83 days old at the start of the experiment, were maintained at $85 \%$ of freefeeding body weight through controlled access to Purina Laboratory Chow and water.

\begin{abstract}
Apparatus
Two standard conditioning chambers housed in sound- and light-attenuating cubicles were each equipped with a retractable response lever located on one wall, a houselight located on the back of the cubicle, a small loudspeaker, a pellet feeder, and a solenoid-operated water dispenser. The pellet feeder dispensed 45-mg food pellets (Noyes, Formula A) into a trough located approximately $85 \mathrm{~mm}$ to the right of the lever. The water dispenser delivered approximately $.1 \mathrm{ml}$ of water per reinforcement into the same trough. Ambient white noise in the room housing the cham. bers masked the sound of control and recording equipment in another room.
\end{abstract}

\section{Procedure}

Instrumental training. Initially, the subjects were given three 15-min adaptation sessions with the houselight on, lever retracted, and several food pellets in the trough at the start of each session. This was followed by 4 days of magazine training during which 36 reinforcers were delivered at varying intervals averaging $25 \mathrm{sec}$. Twelve single-pellet (low-magnitude), 12 six-pellet (highmagnitude), and 12 water reinforcers, all randomly intermixed, were presented.

Finally, during the next three sessions, the lever was continuously present in the chamber from the start of the session with the houselight on. Each press resulted in the delivery of one of the rewards. Twenty-two low-magnitude, 22 high-magnitude, and 22 water rewards, all randomly intermixed, were delivered. Immediately following the 66 th rewarded trial, the lever was retracted and the houselight extinguished.

Pairing phase. During the classical pairing phase, the houselight was on and high-magnitude, low-magnitude, and water reinforcers were presented 12 times each per session to all subjects on a randomly intermixed schedule at intervals ranging from 9 to $39 \mathrm{sec}$, averaging $25 \mathrm{sec}$. For one group (high-manitude paired), a $\mathrm{CS}+$ was presented for $3 \mathrm{sec}$ immediately prior to the delivery of all high-magnitude reinforcements; for the second group (lowmagnitude paired), the $\mathrm{CS}+$ preceded presentations of lowmagnitude reinforcement; and for the third group (water paired), the CS + preceded each presentation of water. For all groups, a $3-\sec$ CS - was also presented 12 times per session, but it was followed by no stimulus presentations for at least $9 \mathrm{sec}$. For half the subjects in each group, the $\mathrm{CS}+$ consisted of a tone $(\sim 1,000 \mathrm{~Hz})$ and the CS - was a click ( $\sim 6$ clicks $/ \mathrm{sec})$. For the remainder, the tone was the $\mathrm{CS}-$ and the click the $\mathrm{CS}+$. There were 18 such classical conditioning sessions.

Discriminative training phase. During this baseline training phase, the response lever was again available and the subjects trained on a discrimination. At varying intervals, averaging $45 \mathrm{sec}$ 
and ranging from 9 to $90 \mathrm{sec}$, the houselight was turned on; the first press of the lever during this $S^{D}$ was followed by a reward and extinguished the houselight. Any leverpress within $12 \mathrm{sec}$ prior to the onset of the houselight postponed its onset for an additional $12 \mathrm{sec}$. A trial was terminated after $60 \mathrm{sec}$ if no leverpress occurred. Twenty trials per session were presented and seven sessions were conducted. Half the subjects in each subgroup received the high-magnitude food reward and the others received the lowmagnitude food reward.

Transfer of control test phase. During the transfer test phase, the tone or click CS was compounded with the light $\mathrm{S}^{\mathrm{D}}$ on Trials 5 , 10,15 , and 20 . These test trials never terminated in a reward during this phase. The tone and click trials were presented alternately and the stimulus presented during the first test trial also alternated from session to session. For half the subjects, the tone was presented first on the 1st day of testing and for the other subjects, the click was presented first. For purposes of comparison, two light-only control trials ( 8 and 18 ) were also scored as test control trials (CON) except that the scheduled reward was delivered. Testing continued for 12 sessions. The time from the onset of the houselight to the first leverpress was recorded for all six test trials.

\section{RESULTS}

\section{Discriminative Training Phase}

The mean response latency of Trials 5 and 15 , the mean of Trials 10 and 20 , and the mean of Trials 8 and 18 were subjected to a mixed analysis of variance. This resulted in three means from each session which corresponded to the conditions of the transfer phase. The between-subject factors were the reward for instrumental baseline responding (low- vs. highmagnitude) and reinforcer signaled by the $\mathrm{CS}+$ in the pairing procedure (low-magnitude food, highmagnitude food, and water). The within-subject factors were trials and sessions. The .05 level of significance was used in all statistical tests.

As might be expected, the instrumental discrimination was acquired fastest by the animals receiving high-magnitude reinforcement. This was reflected in a significant main effect of reinforcer for the instrumental baseline $[\mathrm{F}(1,42)=6.93, \mathrm{p}<.05]$ and a significant Baseline by Session interaction $[F(6,252)$ $=4.97, \mathrm{p}<.001]$. The overall effect of sessions was also highly significant $[\mathrm{F}(6,252)=23.47, \mathrm{p}<.001]$. A separate analysis of the last session of baseline training showed no statistically significant effects, reflecting the decline of the differential effect of the instrumental reinforcer across sessions.

\section{Transfer of Control Test Phase}

The means of the two trials for each CS contingency $(\mathrm{CS}+. \mathrm{CS}-$, and $\mathrm{CON})$ for each test session were also subjected to a mixed analysis of variance: between group effects were pairing procedure (lowmagnitude food, high-magnitude food, and water paired) and baseline reward (low-vs. high-magnitude food). The within-subject effects were CS contingency $(\mathrm{CS}+, \mathrm{CS}-$, and $\mathrm{CON})$ and sessions. Only the first four sessions were analyzed because the effects of the CSs had extinguished by the fifth session. The mean latency to the auditory stimuli plus houselight and houselight alone (control trials) for each of the six groups are shown in Figure 1. Generally, the effects of the CSs differed across groups, which was reflected by a significant Pairing Procedure by Baseline by CS Contingency interaction $[F(4,84)=$ $7.91, \mathrm{p}<.001]$.

One specific prediction derived from an associative model of transfer was that subjects receiving a lowmagnitude reward would be more disrupted by a $\mathrm{CS}+$ paired with a high-magnitude reinforcer than by a $\mathrm{CS}+$ paired with a low-magnitude reinforcer. This prediction was confirmed by comparing the amounts of disruption (CS + minus $\mathrm{CS}-$ ) between the high-magnitude paired and low-magnitude paired groups receiving the low-magnitude reward $[F(1,126)$ $=23.09, \mathrm{p}<.001]$. However, the complementary prediction that subjects receiving the high-magnitude reward would be more disrupted by a $\mathrm{CS}+$ paired with a low-magnitude reinforcer did not receive statistical confirmation $[\mathrm{F}(1,126)=.57, \mathrm{p}>.10]$.

To test whether the water-paired CSs significantly disrupted the food-rewarded response, the two WAP groups were combined and a test of the difference between the CS + and CS - stimuli was conducted. This test revealed that the CSs for water significantly disrupted the food-rewarded baselines $[F(1,126)$ $=5.77, \mathrm{p}<.05]$.

The disruptive effect of the CS + attributable to its reinforcer signaling properties is revealed by the contrasts between mean latencies to the $\mathrm{CS}+$ and $\mathrm{CS}-$ within each group, which provides potentially useful information orthogonal to the previous contrasts. They showed that, for the low-magnitude baseline groups, the high-magnitude-paired CS + differed significantly from its $C S-$ control $[F(1,126)=$

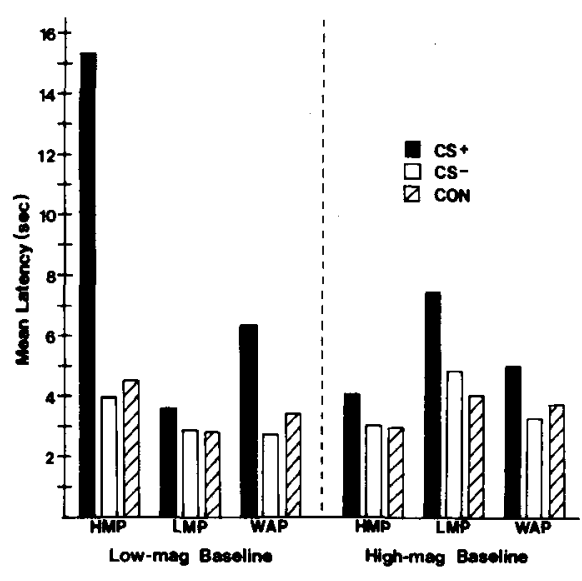

Figure 1. Mean latencies of test trials for the low-magnitude paired (LMP), high-magnitude paired (HMP), and water-paired (WAP) groups receiving low-magnitude (low-mag) reward and highmagnitude (high-mag) reward. 
$52.45, \mathrm{p}<.001]$ and the $\mathrm{CS}+$ for water differed significantly from its control $[F(1,126)=5.19, p<$ $.05]$. None of the six remaining differences was statistically significant.

This pattern of effects was also observed for the first session alone and held under a variety of transformations (e.g., logarithmic, reciprocal, CS + minus CS - differences), indicating that the pattern is quite stable.

\section{DISCUSSION}

The specific predictions of an associative model of transfer were only partially supported by the present results. A CS + for high-magnitude food $\left(\mathrm{CS}_{h i}^{+}\right)$ disrupted a low-magnitude baseline significantly more than a $\mathrm{CS}+$ for low-magnitude food $\left(\mathrm{CS}_{\mathrm{lo}_{0}}^{+}\right)$. This effect is consistent with previous research (Capaldi et al., 1976; Carlson, 1976) and has usually been taken as strong evidence in support of an associative view of transfer of control. Since, in this case, the baseline response would be under the stimulus control of a mediator associated with one pellet, an associative view predicts the $\mathrm{CS}_{\mathrm{hi}}^{+}$would disrupt responding, because the mediator it elicits is different from the mediator associated with the instrumental response. This same result contradicts an incentive motivational view because $\mathrm{CS}_{\mathrm{hi}}^{+}$would, according to such a theory, be expected to elicit more motivation (or $\mathrm{K}$ ) than $\mathrm{CS}_{\mathrm{l}_{0}}^{+}$such that the latencies of $\mathrm{CS}_{\mathrm{hi}}^{+}$would be the shortest.

On the other hand, the effects of the food $\mathrm{CS}+\mathrm{s}$ upon the response for a high-magnitude reward in the present study were not completely consistent with an associative model of transfer. Although $\mathrm{CS}_{\mathrm{lo}}^{+}$ disrupted the high-magnitude baseline somewhat more than $\mathrm{CS}_{\mathrm{hi}}^{+}$, as predicted by associative theory, the effect was not statistically reliable. However, with respect to the viability of an associative account vs. a motivational account, this effect is neutral, since both models generate the same, erroneous prediction.

Consistent with predictions derived from the associative model, $\mathrm{CS}+\mathrm{s}$ for water significantly disrupted the food-rewarded baselines, although the difference was smaller than expected. The small difference might indicate that the mediators conditioned by water and food may not have differed substantially on some dimension orthogonal to their physical properties. It is also possible, as noted below, that mediators for food and water can exist independently, so that CSs paired with water do not cause large changes in baseline behavior rewarded with food. This problem must remain open to additional research. However, the relative advantage of the associative model over the motivational model remains, because the motivational model could not predict the relative effect of the food and water CSs without prior data concerning the amounts of motivation they elicit.

In all conditions of the present experiment, the $C S-s$ had almost no effect upon the baseline behavior. This might appear to contrast with some findings that a CS - can show inhibitory effects (e.g., Hyde et al., 1968; Trapold \& Winokur, 1967). However, in the present experiment, the procedure tended to counter such effects. First, during testing, the $\mathrm{CS}-\mathrm{s}$, like the $\mathrm{CS}+\mathrm{s}$, were compounded with the previously trained discriminative stimulus for the baseline response. In addition, the pairing procedure allowed each $\mathrm{CS}$ - to occur in close temporal proximity to the two reinforcers which were not paired with the CS + . The net result was to reduce the effects of the CS $-\mathrm{s}$ upon the baseline behavior.

With respect to the overall pattern of CS + effects it may be that an associative model of transfer, while basically correct, requires supplementation by other principles recognizing the inherent stability of strongly reinforced behaviors. Perhaps a high-magnitude baseline, with its greater habit strength, is simply less disruptable than a low-magnitude baseline. The larger (but nonsignificant) disruption caused by the $\mathrm{CS}+$ for water on the low-magnitude baseline compared to its effect on the high-magnitude baseline partially supports this suggestion. It is also conceivable that the low-magnitude mediator would be masked by the presence of the high-magnitude mediator elicited by the baseline discriminative stimulus. The combined mediator would then exhibit properties not very different from the high-magnitude mediator alone. This "mediator salience" principle would also allow for the strong associative effect obtained with a low-magnitude baseline and might also be extended to the effects of the water $\mathrm{CS}+\mathrm{s}$ if it is assumed that the mediators associated with water and food can occur independently.

Capaldi et al. (1976) offered an alternative account of transfer which assigns two properties to a CS +. In addition to its associative effects, they assumed that a $\mathrm{CS}+$ would tend to produce frustration and/or inhibition, if a response was rewarded with a reinforcer smaller than expected in the presence of the $\mathrm{CS}+$. In the present experiment, subjects were never rewarded in the presence of the CS + $\mathrm{s}$ during testing. Thus, different amounts of frustration might have been elicited during the $\mathrm{CS}+\mathrm{s}$. Considering only the food-CS $+\mathrm{s}, \mathrm{CS}_{\mathrm{hi}}^{+}$might have elicited more frustration than $\mathrm{CS}_{\mathrm{lo}}^{+}$so that the better performance to $\mathrm{CS}_{\mathrm{lo}}^{+}$ would have been expected. Under the low-magnitude baseline, frustration effects would have been compatible with the associative effects of the CS+s. However, under the high-magnitude baseline, frustration effects would have opposed the expected associative effects. This process might explain why the effects of the CS $+s$ were not symmetrical. 
Although supplementing the associative model with the frustration account proposed by Capaldi et al. might appear to provide a neat explanation of transfer effects, it is not without problems. First, the pattern of CS effects on the 1st day of testing was nearly identical to the pattern shown by all 4 test days combined. This is not consistent with a frustration account, because interference with responding should have been delayed until an anticipation of frustration was conditioned to the discriminative stimulus-CS + compound. In the presence of the rather difficult discrimination required of the animals (CS + plus $S^{D}$ vs. $S^{D}$ alone), this process might be expected to extend over more than the eight test trials to which each animal was subjected. More information concerning the development of frustration under these particular circumstances is necessary before it is presumed that such a process is involved in transfer phenomena.

In conclusion, associative theory seems to account best for the present data, although it does require additional assumptions. On the other hand, the relative effects of $\mathrm{CS}_{\mathrm{hi}}^{+}$and $\mathrm{CS}_{\mathrm{lo}}^{+}$upon the low-magnitude baseline are simply not compatible with a motivational account. Therefore, further elaboration of the associative model might prove the most fruitful course for additional transfer of control research.

\section{REFERENCES}

Capaldi, E. D., Hovancix, J. R., \& Friedman, F. Effects of expectancies of different reward magnitudes in transfer from noncontingent pairings to instrumental performance. Learning and Motivation, 1976, 7, 197-210.

Carlson, J. G. Effect of a stimulus paired with reinforcement as a function of reinforcement magnitude. Bulletin of the Psychonomic Society, 1976, 7, 254-256.

Hyde, T. S., Trapold, M. A., \& Gross, D. M. Facilitative effect of a CS for reinforcement upon instrumental responding as a function of reinforcement magnitude: $A$ test of incentive motivation theory. Journal of Experimental Psychology, 1968, $73,423-428$.

Rescort., R. A., \& Solomon, R. L. Two-process learning theory: Relationships between Pavlovian conditioning and instrumental learning. Psychological Review, 1967, 74, 151-182.

SpEnCE, K. W. Behavior theory and conditioning. New Haven: Yale University Press, 1956.

Trapold, M. A., \& Overmier, J. B. The second learning process in instrumental learning. In A. H. Black \& W. F. Prokasy (Eds.), Classical conditioning II. New York: Appleton-CenturyCrofts, 1972.

Trapold, M. A., \& Winokur, S. Transfer from classical conditioning and extinction to acquisition, extinction, and stimulus generalization of a positively reinforced instrumental response. Journal of Experimental Psychology, 1967, 73, 517-525.

(Received for publication March 31, 1978; revision accepted October 24,1978 .) 